\title{
Type-2 Fuzzy Logic System Applied to a Temperature Control of an Electric Oven
}

\author{
Leonardo A. Serapião* Eduardo R. C. Queiroz ** \\ Alexandre B. dos Santos ${ }^{* * *}$ Thiago V. N. Coelho ${ }^{* * * *}$ \\ Daniel D. Silveira ${ }^{\dagger}$ Eduardo P. de Aguiar ${ }^{\ddagger}$ \\ * Department of Industrial and Mechanical Engineering, Federal \\ University of Juiz de Fora, MG (leonardo.serapiao@engenharia.ufj..br) \\ ** Graduate Program in Industrial Engineering, Pontifical Catholic \\ University of Rio de Janeiro, RJ (eduardo.campos@engenharia.ufj..br) \\ *** Department of Electrical Engineering, Federal University of Juiz de \\ Fora, MG (alexandre.bessa@engenharia.ufjf.br) \\ **** Department of Electrical Engineering, Federal University of Juiz \\ de Fora, MG (thiago.coelho@ufjf.edu.br) \\ ${ }^{\dagger}$ Department of Electrical Engineering, Federal University of Juiz de \\ Fora, MG (danieldsilveira@gmail.com) \\ $¥$ Department of Industrial and Mechanical Engineering, Federal \\ University of Juiz de Fora, MG (eduardo.aguiar@engenharia.ufjf.br)
}

\begin{abstract}
:
The control of non-linear systems is a difficult task, requiring efficient control algorithms and good accuracy. The control of these processes requires a good ability to deal with uncertainties, essentially caused by changes in the environment of the system. To address this problem, a prototype oven was built, connected to a PC through embedded electronics, allowing the test and validation of three different control algorithms. A comparison was done among the proposed models: rule-based control algorithm, type-1 fuzzy logic system, and type-2 fuzzy logic system. Also, it is analyzed their effectiveness based on their performance and ability to deal with uncertainties. Experimental results show that the type-2 fuzzy logic system has a better response having an improved rise time, minimum overshoot and the best performance. It also showed a continuous response, with a better approximation of the desired result, and an excellent ability to handle with uncertainties.
\end{abstract}

Keywords:

Type-2 fuzzy logic system. Type-1 fuzzy logic system. Temperature Control. Electric Oven.

\section{INTRODUCTION}

Systems that work with heating or cooling cycles are required in many practical applications, e.g. ovens, furnaces and air conditioning. The objective of temperature control is to heat up a system to a limit, and maintain the temperature as long as necessary. In this period, only small variations are accepted, characterizing a stable process of heat transfer between oven and system being heated. This is normally achieved by maintaining the temperature constant all along the process and useful lifetime of the oven or furnace, independent of ambient variations, like room temperature, mains voltage fluctuations, position of the oven and other heat sources which can interfere at this control. Focusing on electric ovens, it is a difficult task to have good accuracy in the set point temperature, especially caused by the inefficiency of oven insulation and environment disturbances. Achieving maximum accuracy in the system is still a challenge.

Studies modeled this kind of control as nonlinear systems, where the input is the heating element and the output is the temperature inside the oven. The temperature control of an electric oven with electric resistances generally can be done by means of variation of two parameters: phase angle of AC mains or sending a finite number of $\mathrm{AC}$ cycles, switching on and off the resistances in a short period of time. This can be accomplished by a pulsewidth modulation signal. This methodology has shown to be effective, and can be used with several methods, as PID control, fuzzy rule based system and smith predictor methods, or with multiple procedures like those combined.

Those systems are, however, sensible to random disturbances. Those disturbances lead the control network to send inappropriate signals to the heating control process, considering a state that is no longer real. A simple way to characterize external disturbances is to place a sensor outside the oven. This data is then also send to the controller, which includes the external temperature in the calculations.

Another challenge for controlling this type of system is the considerable time between the control signal sent by the controller (a computer) and the response of system. Called long dead-time process, these plants do not present 
an immediate response to the commands of the controller, so that different methods are developed to accomplish this control, using innovative tuning parameters or compensators.

Based on the above challenges to design a proper controller, the main objective of this work is to present a control system which can be used in any oven, addressing all problems stated above, and also considering the modification of a few factors, such as method of heat transfer, operating voltage, temperature range and ambient conditions, that could change for different ovens. This controller shall perform well because it does not consider constructive information of the oven, presenting good efficiency on temperature control Lima et al. (2015) and joining the best qualities of several studies in this field, as its architecture just considers variations of temperature inside and outside the oven.

To allow the comparison of different controllers, it was developed a specific routine, presented in Figure 1, designed to improve the response time and the efficiency of the system. The communication with the computer was accomplished using a serial port interface. The control loop initiates defining a set-point temperature. Then, the controller defines the duty-cycle of the PWM, based on the temperature measured by LM35 sensors. Finally, the loop repeats until the set-point is reached.

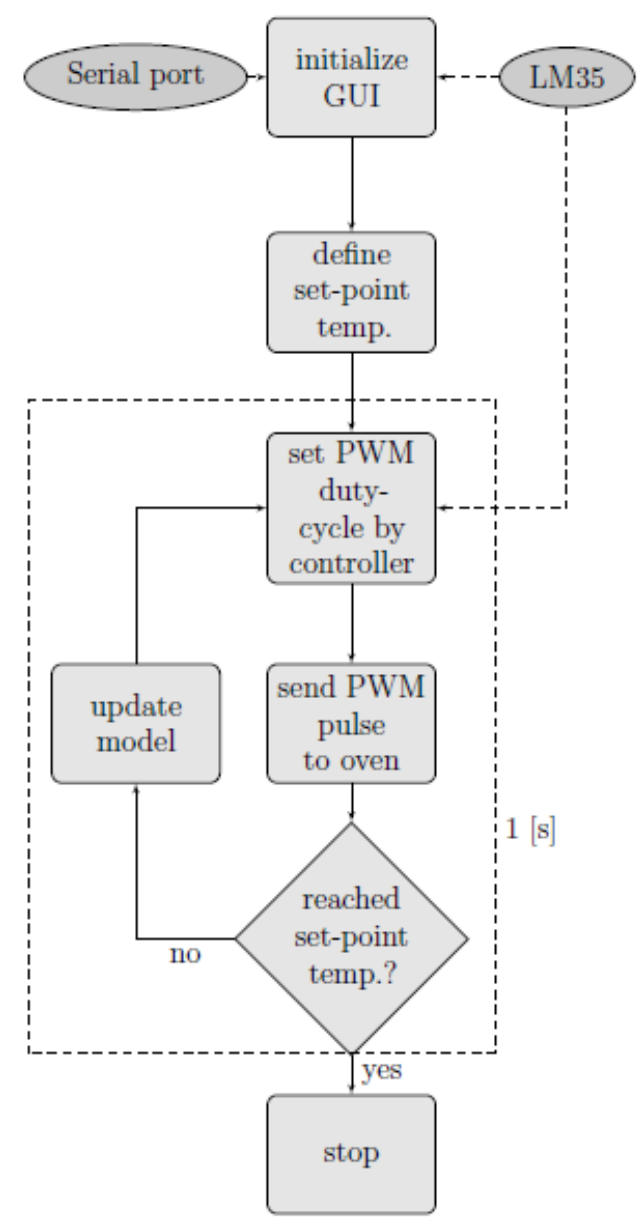

Figure 1. Model flowchart
The strategy chosen to design the final controller was to combine fuzzy logic and an embedded micro processed hardware. Also like in Singhala et al. (2014), effort was done in order to implement this idea using low cost hardware, so it could be used in different kinds of heating plants, such as industrial ones and home appliance.

The control plant has to be able to normalize random disturbances, to predict the behavior of the system being controlled, and also to analyze possible variations and to make decisions based on a pre programmed code. This controller optimizes the process to the best output as possible. Type-1 FLSs have been massively applied aiming to solve the problem of oven temperature control de Aguiar et al. (2017). Although type-1 FLSs offer a reasonable performance, some uncertainties associated with the control problem is something that type-1 FLS may not properly handle. According to Mendel (1938), the type-2 FLS have the potential to handle larger uncertainties than a type-1, therefore it has the ability to adjust to the variations that the system may suffer.

This work proposes then a comparison between type- 1 and type-2 FLSs de Aguiar et al. (2018) control algorithms, and also an ordinary rule-based control algorithm, in order to define the most appropriate controller to handle large uncertainties in the experimented system, and achieve the maximum accuracy and efficiency.

Major expected conclusions are as follows:

- Type-2 FLS has more precisely convergence rate, eliminating disturbances effects on main proposal and less variation on temperature;

- Low cost hardware interface, using simple electronic components, such that the oven with this additional hardware does not have a price higher than its benefits.

The remaining of the paper is organized as follows: Section II aims to discuss details of the setup adopted. Section III discusses the controllers adopted. Section IV discusses the results of the tests. Section V states the main conclusions regarding the proposals.

\section{MEASUREMENT SETUP}

The measurement setup is composed by a prototype oven and a embedded electronic board connected to a PC running Matlab, allowing the control of the oven temperature by means of the duty cycle of a PWM signal. Those components will be detailed in this section.

\subsection{Experimental Oven}

The ordinary electric oven chosen for the experiment and measurements has two independent resistances, one at the top and one at the bottom. Each one consumes a maximum power of $750 \mathrm{~W}$, resulting in a final total power of 1500 W.

A prototype oven was then built based on this oven to allow the control of the amount of power delivered to each resistance. This experimental oven was built with high temperature resistant silicon wiring for power supply and data collection of these sensors. The temperature is 
measured by six LM35 temperature sensors, one in the middle (for very accurate temperature measurements of an object under test), one at the front door, one in the back, and two located on both sides of the oven. Another sensor is located outside the oven for ambient temperature measurement. They are also rated for full $-55^{\circ} \mathrm{C}$ to $150^{\circ} \mathrm{C}$ range, without need of external calibration, having an accuracy of $0.5^{\circ} \mathrm{C}$ at $25^{\circ} \mathrm{C}$. A picture of the oven with sensors is shown in Figure 2.

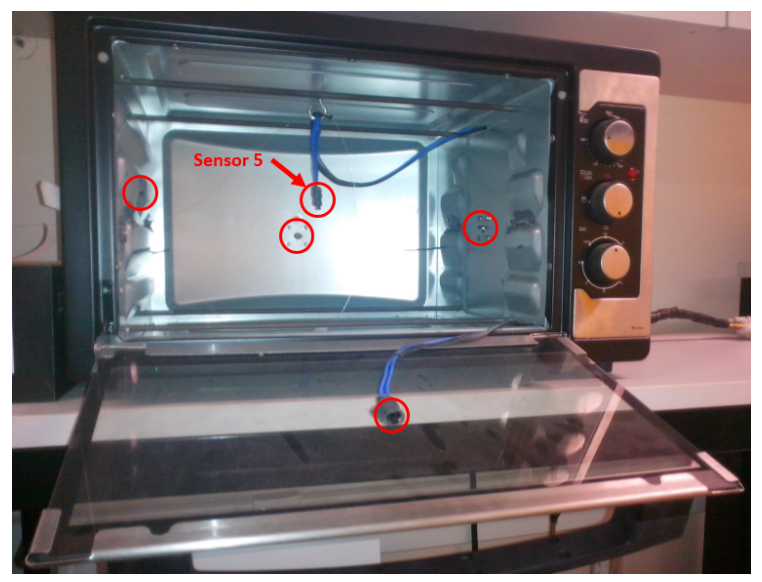

Figure 2. Oven with sensors

\subsection{Embedded Electronic Board}

The control of the temperature of the oven was made possible by the variation of the duty cycle of a pulse width modulation (PWM) signal, practically an on-off configuration. Among many types of converters, the PWM has the better response to deal with on-off power supply switching Tzou et al. (1993). Although the control of the pulse width is very precise, this method adds uncertainty about the actual value of temperature, if used in an open loop configuration. So it was necessary not only generate the PWM signal with variable duty cycle to control the oven, but also to collect the actual temperature precisely, this way enabling the closed loop control of the oven.

An embedded control board was then built, having an MSP430G2553 microcontroller that receives commands from the PC and generate PWM pulses to drive the zerocross phototriac driver optocouplers (MOC3063).

This square wave PWM control signal has a frequency of 3 $\mathrm{Hz}$, with a variable duty cycle defined by the user. These pulses will determine the amount of power delivered by the high current Triacs (TIC246) to the resistances of the oven.

The microcontroller is also programmed to collect the data generated by the LM35 sensors, using its internal 10 bits ADC. It transmits this vector of data to the PC as required. The control software running at the $\mathrm{PC}$ was configured to collect sensor temperature information every second. A synthesis of all elements of the control system is presented in Figure 3.

Then, from a fixed frequency and variable duty cycle signal of the microcontroller, embedded circuits optimally switch the $60 \mathrm{~Hz}$ sine wave from the energy provider for resistances located at the top and at the bottom of the

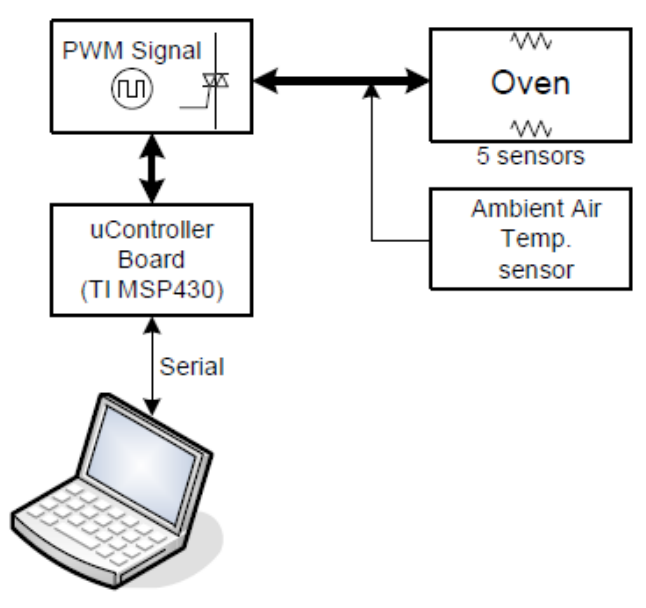

Figure 3. Synthesis of the embedded electronic board oven, converging to a dedicated interface board for signal conditioning composed of regulators, LM358 opamps, and protection circuits, as shown in Figure 4.

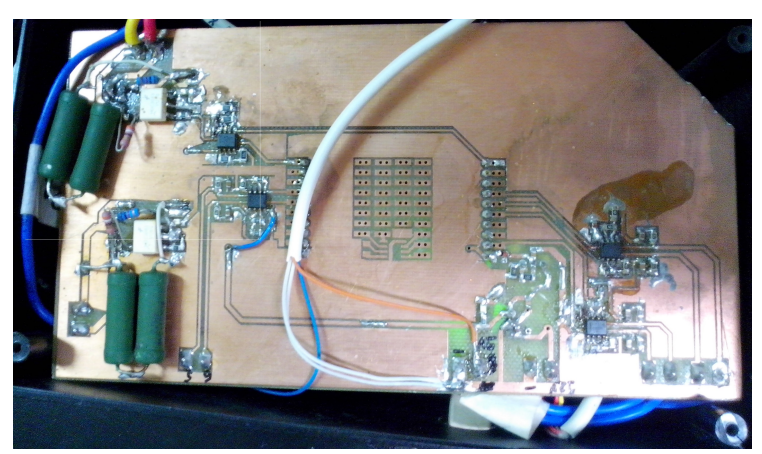

Figure 4. Data colletion and power transmission board

The processing software was built in MATLAB graphical user interface (GUI).

Analysis of PWM pulse The selected method of control of the oven temperature was by varying the duty-cycle of a PWM pulse. The input of the MOC3063 requires a low state to turn the TRIAC on, so a $15 \%$ duty cycle means that the TRIAC is on at $85 \%$ of the time, as shown in the figures.

It is desirable to know with a good precision the relation of the duty-cycle signal of the PWM needed to heat up the system and also to stabilize the internal temperature of the oven in a given temperature. In order to achieve this objective, different pulses were generated and the temperature achieved was analyzed. After numerous tests, it was possible to generate a graph that shows the percentage of the duty-cycle needed to stabilize at different temperatures. This relation is presented in Figure 5.

\subsection{Equipment set-up and boundary conditions}

To ensure the highest accuracy possible in the experiment, few conditions were established:

- The ambient temperature was set in $28^{\circ} \mathrm{C}$ controlled by an air conditioner, in all trials, to secure no external temperature disturbances; 
Pulse Needed to Stabilization

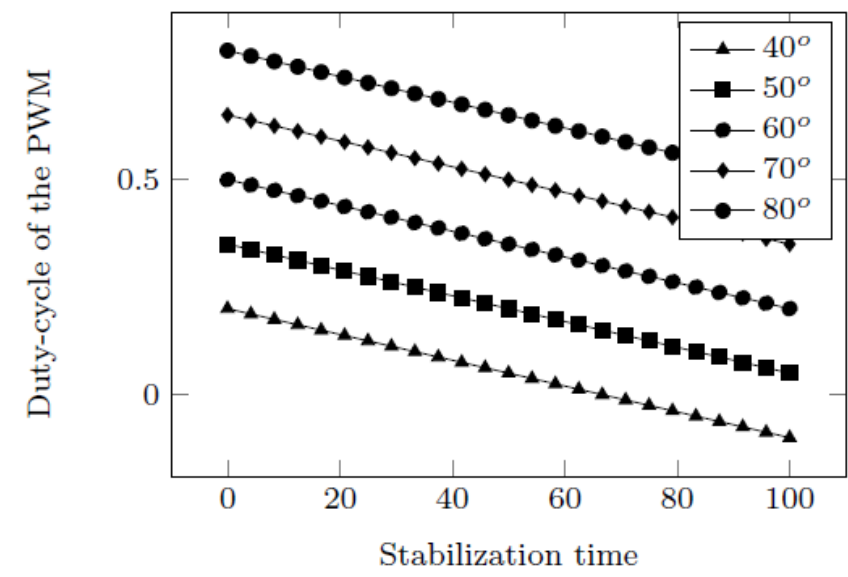

Figure 5. Relation between temperature and the duty-cycle of the PWM

- The oven was preheated at $30^{\circ} \mathrm{C}$ to guarantee an uniform temperature distribution in entire oven;

- The heating process was defined with two stages of warming and stabilization, with temperatures of $50^{\circ} \mathrm{C}$ and $60^{\circ} \mathrm{C}$;

- Restricted use of computer to data collection system, to ensure that all processing is available to the software, where the data collection is performed at frequency of $1 \mathrm{~Hz}$.

Table 1 presents the correction factors that were heuristically defined in order to adjust the duty-cycle of the PWM regarding the ambient temperature. In this work, as we set the ambient temperature in $28^{\circ} \mathrm{C}$, the correction factor adopted was equal to 0.92. Thus, the output of the controller is multiplied by those factor.

Table 1. Environment Temperature Corrections

\begin{tabular}{cc} 
Temperature Range $\left({ }^{\circ} \mathrm{C}\right)$ & Correction Factor \\
\hline $15-20$ & 1.00 \\
$20-25$ & 0.96 \\
$25-30$ & 0.92 \\
$30-35$ & 0.88 \\
\hline
\end{tabular}

\section{CONTROL DESIGN}

The objective of the controller in this work is to perform the control of the output temperature of an electric oven, $T_{\text {out }}\left({ }^{\circ} \mathrm{C}\right)$, as a function of duty-cycle of the PWM. The Figure 6 presents the closed-loop control system proposed. To establish efficient results, three control systems have been proposed.

\subsection{Rule-based control algorithm}

The first one presents a rule-based control algorithm (RBCA), as seen in Algorithm 1. This algorithm runs according two main steps:

- To define the temperature ranges equally divided respecting the oven maximum and minimum temperatures, collecting the instantaneous temperature at the center of the oven, $T_{\text {out }}$, and calculates the error, $\Delta E\left({ }^{\circ} \mathrm{C}\right)=T_{\text {set }}-T_{\text {out }}$

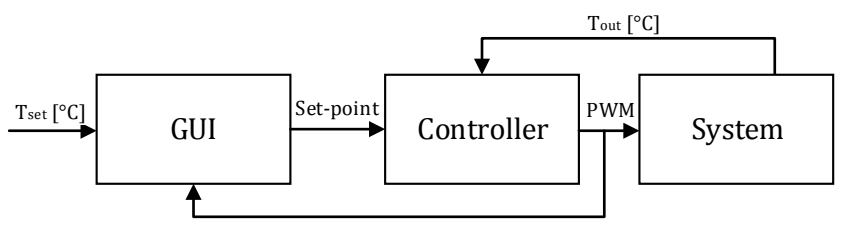

Figure 6. Control system diagram

- Using this information, the algorithm defines the limits between $l_{1}$ and $l_{4}$, in order to determine the percentage of the duty cycle of the PWM.

This cycle repeats at a frequency of $1 \mathrm{~Hz}$.

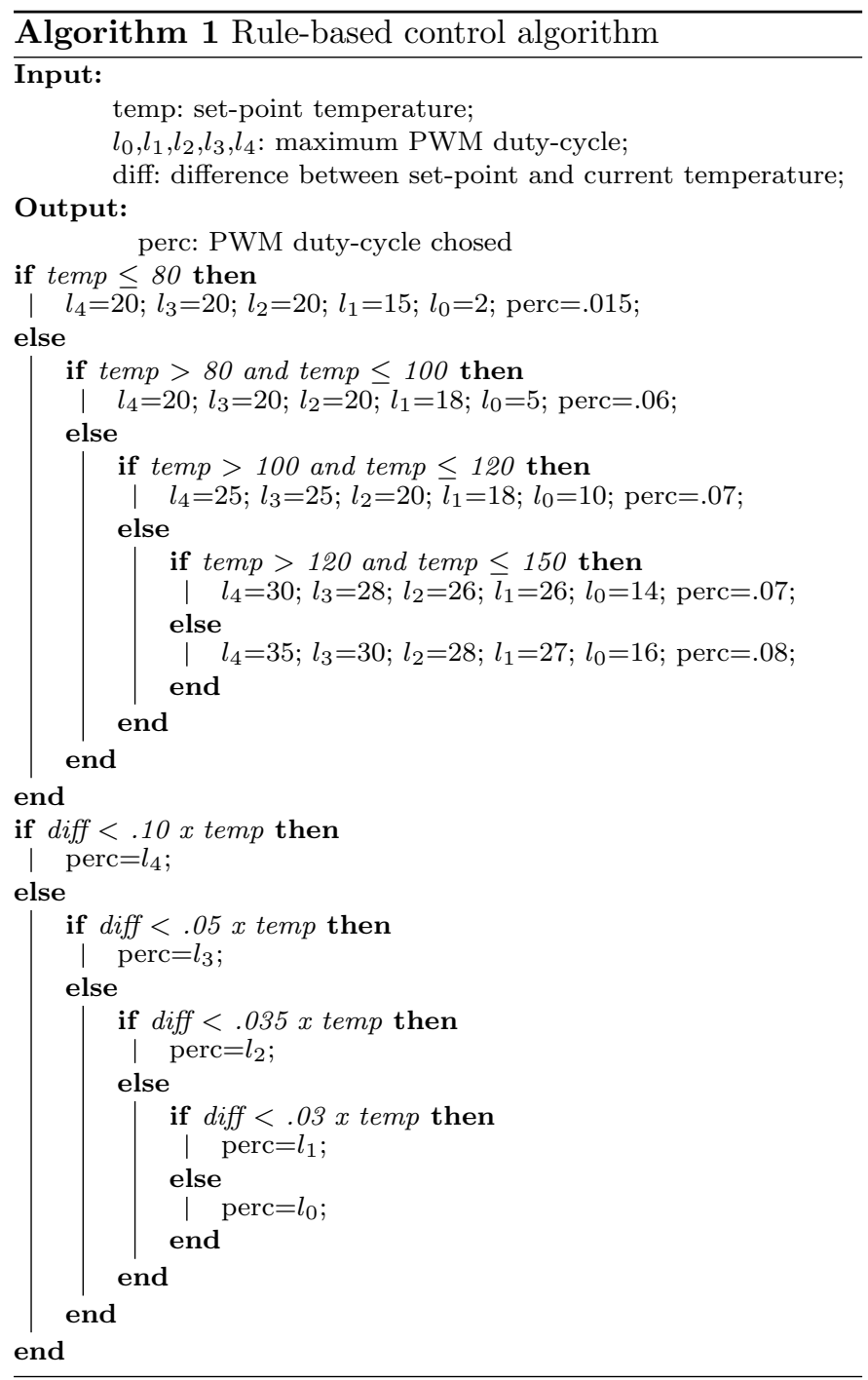

\subsection{FLSs control algorithms}

The two other controllers are FLSs control algorithms, respectively type-1 fuzzy logic controller (T1FLC) and type2 fuzzy logic controller (T2FLC). The T1FLC is chosen considering the best performance to handle uncertainties, 
associate with non-linear response of the oven, i.e. the heating process is described by differential equations.

The inference method was determined as Takagi-Sugeno fuzzy model. Numerous results on stability analysis, nonlinear control and controller synthesis have been developed for those models. Two inputs were selected. $T_{\text {out }}$ is temperature in sensor five, which represents the center of the oven, and discriminates stabilization (ST), small (SM), medium (ME), big (BG) and very big (VB). $\Delta E\left({ }^{\circ} \mathrm{C}\right)$ is the difference between the set point and actual temperature, and discriminates medium negative $(\mathrm{MN})$, small negative $(\mathrm{SN})$, zero $(\mathrm{ZO})$, small positive $(\mathrm{SP})$ and medium positive (MP).

The output selected is the duty-cycle of the PWM, \% $\mathrm{P}$, and discriminates stabilization (ST), small (SM), medium (ME), big (BG) and very-big (VB). Table 2 presents the decision matrix to compute the values of $\% \mathrm{P}$.

We consider singleton fuzzification, max-product composition, product implication, centroid defuzzifier and triangular membership functions.

The input variables $T_{\text {out }}, \Delta E$ and the output variable $\% \mathrm{P}$ are shown in Figures 7, 8 and 9, respectively. The corresponding ranges are $[20,80],[-37.5,37.5],[0,24]$ Celsius degrees for $T_{\text {out }}$ and $\Delta E$, and percentage for $\% \mathrm{P}$, respectively.

The T2FLC has a potential to handle dynamic uncertainties Farooq et al. (2013). Researchers have been worked in recent years towards demonstrating better properties of the T2FLC over the T1FLC.

Table 2. Decision Matrix

\begin{tabular}{cc} 
Temperature Range $\left({ }^{\circ} \mathrm{C}\right)$ & Correction Factor \\
\hline $15-20$ & 1.00 \\
$20-25$ & 0.96 \\
$25-30$ & 0.92 \\
$30-35$ & 0.88 \\
\hline
\end{tabular}

In this work the T2FLC and T1FLC have the same input and output variables and MFs ranges. For T2FLC, we defined gaussian membership functions for $T_{\text {out }}$ and $\Delta E$, as presented in Figures 10 and $11 . \% \mathrm{P}$ presents $\mathrm{MF}$ with constant values. T2FLC presents equivalent sets according as defined for T1FLC. The mean and standard deviation for each MF of T2FLC are described in Table 3, where $\mid m$, $m|,| \sigma$ and $\sigma \mid$ are Left Mean, Right Mean, Left Deviation and Right Deviation, respectively.

Table 3. T2FLC MF parameters

\begin{tabular}{ccccc} 
Variable & $\mid m$ & $m \mid$ & $\mid \sigma$ & $\sigma \mid$ \\
\hline$T_{\text {out }}$ & {$[28,38,48,58,68]$} & {$[32,42,52,62,72]$} & 4 & 4 \\
$\Delta E$ & {$[-22.5,-10,-2.5,10,22.5]$} & {$[-27.5,-15,2.5,15,27.5]$} & 4 & 4 \\
\hline
\end{tabular}

We chose center of gravity as the method of defuzzification and type-reducing Karnik and Mendel (2001). All T2FLC operators are equivalent to T1FLC.

\section{EXPERIMENTAL RESULTS}

In order to compare the control systems, each test was performed three times, the average response was saved in a data file. Every attempt was made following the boundary

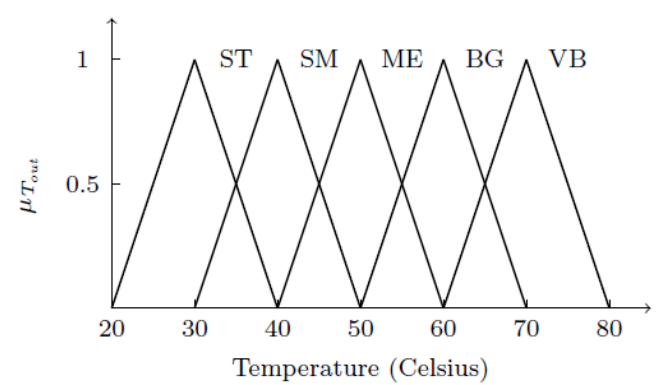

Figure 7. Type-1 MF for $T_{\text {out }}$

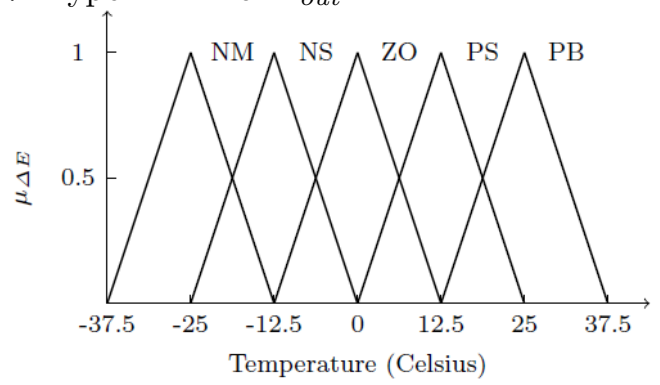

Figure 8. Type-1 MF for $\Delta E$

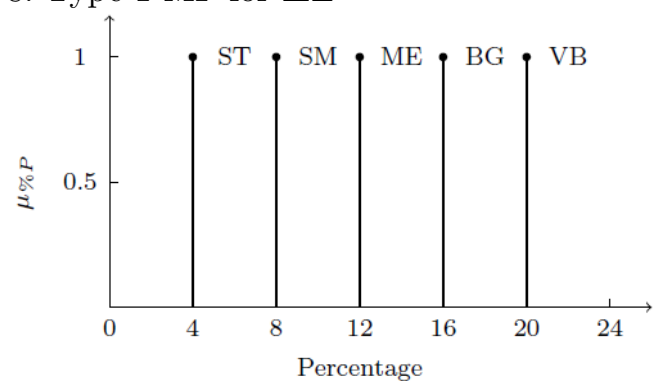

Figure 9. Type-1 MF for $\% \mathrm{P}$

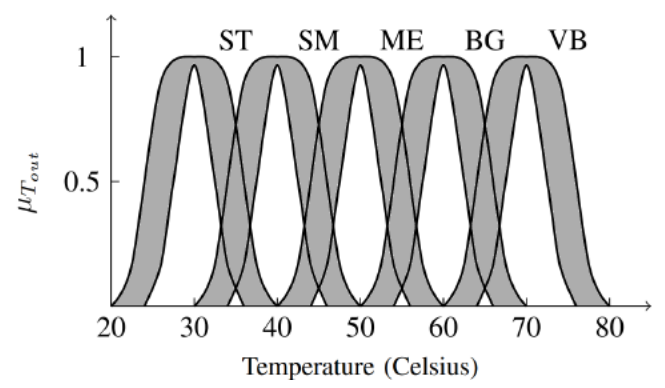

Figure 10. Type-2 MF for $T_{\text {out }}$

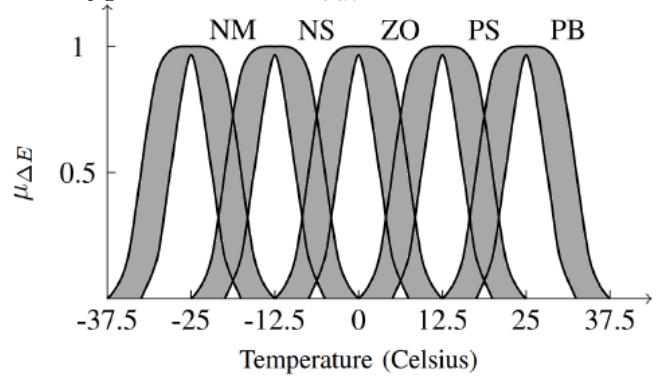

Figure 11. Type-2 MF for $\Delta E$ 
conditions proposed by establishing two temperature levels, $50^{\circ} \mathrm{C}$ and $60^{\circ} \mathrm{C}$. The mean responses of each controller are arranged in Figure 12. Although they are not presented here, the proposed models are consistent and offer similar response for the other temperature levels.

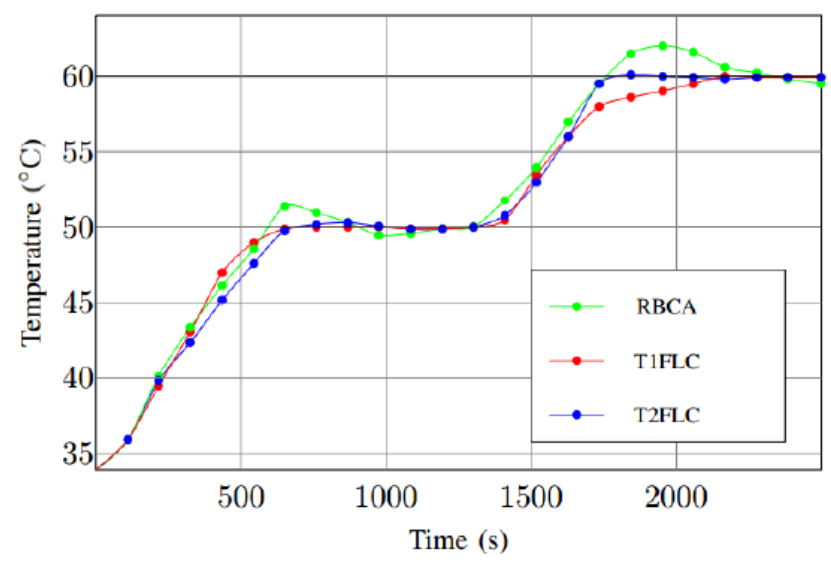

Figure 12. Response of the controllers

Based on the experimental results, it is possible to ensure the optimized configuration for the system, in terms of ascertaining the best rising ramp in order to minimize the percent overshoot. T2FLC presents the better approximation of the desired response, since it had the minimum rise time with the smallest overshoot. Furthermore it is noticed that for higher temperatures, a higher heat input is required, to maintain the stabilization temperature. Moreover, it was observed that even with a minimum pulse, the oven heats continuously, as long is provided an stabilization value, which is established as $5 \%$ of the dutycycle of the PWM.

The three control algorithms accomplished the proposed task, since all reached the temperature set point and showed some stability. However, considering the purpose of the work, which is to maximize performance and improve the ability to deal with uncertainties, RBCA and T1FLC did not show excellent performance.

On the other side, the system operating with T2FLC presents an undeniable accuracy with respect to the uncertainties, having an improved rise time, maximized when compared to other controllers. Although T2FLC presents a larger rise time than RBCA, it also shows minimum overshoot, especially caused by the system that controls the value of the derivative of the rising ramp. Table 4 presents the rise time and the maximum error, occurred in the overshoot, for the two temperature levels experimented.

Table 4. Controllers Experimental Results

\begin{tabular}{cccc} 
Parameter & RBCA & T1FLC & T2FLC \\
\hline Rise Time [s] at $50^{\circ} \mathrm{C}$ & 585 & 612 & 616 \\
Rise Time [s] at $60^{\circ} \mathrm{C}$ & 1736 & 2214 & 1747 \\
Max Error [ ${ }^{\circ} \mathrm{C}$ ] at $50^{\circ} \mathrm{C}$ & 1.9 & 0.2 & 0.1 \\
Max Error [ ${ }^{\circ} \mathrm{C}$ ] at $60^{\circ} \mathrm{C}$ & 2.7 & 0.1 & 0.1 \\
\hline
\end{tabular}

\section{CONCLUSION}

This work presented three different control algorithms, used to perform the control of the temperature of an electric oven. A comparison was done among RBCA, T1FLC and T2FLC, and analyzed their effectiveness based on their performance and ability to deal with uncertainties.

The numerical results, obtained through experiments, showed that the T2FLC has a better response than any other controller, having an improved rise time, minimum overshoot and the best performance compared to the experimented control algorithms. It also showed a continuous response, with a better approximation of the desired result, and an excellent ability to handle uncertainties.

Future work is to improve the controller by addiction of an neural fuzzy control algorithm and also prototype an equipment to be integrated into any kind of ovens, in order to perform the on-line temperature control.

\section{REFERENCES}

de Aguiar, E.P., Amaral, R.P.F., Vellasco, M.M.B.R., and Ribeiro, M.V. (2018). An enhanced singleton type-2 fuzzy logic system for fault classification in a railroad switch machine. Electric Power Systems Research, 158, 195-206.

de Aguiar, E.P., Nogueira, F.M.A., Vellasco, M.M.B.R., and Ribeiro, M.V. (2017). Set-membership type-1 fuzzy logic system applied to fault classification in a switch machine. IEEE Transactions on Intelligent Transportation Systems, 18, 2703-2712.

Farooq, U., Gu, J., and Seto, M. (2013). Design and comparison of takagi-sugeno type-1 and interval type2 fuzzy logic controllers for posture stabilization of wheeled mobile robot. International Conference on Robotics and Biomimetics, 2309-2317.

Karnik, N. and Mendel, J.M. (2001). Centroid of a type-2 fuzzy set. Information Sciences, 132, 195-210.

Lima, D.M., Normey-Rico, J.E., and Santos, T.L.M. (2015). Temperature control in a solar collector field using filtered dynamic matrix control. ISA Transactions, $62,39-49$.

Mendel, J.M. (1938). Uncertain Rule-Based Fuzzy Logic System: Introduction and New Directions. Prentice Hall.

Singhala, P., Shah, D.N., and Patel, B. (2014). Temperature control using fuzzy logic. International Journal of Instrumentation and Control Systems, 4, 1-10.

Tzou, Y.Y., Ho, L.H., and Ou, R.S. (1993). Fuzzy control of a closed-loop regulated PWM inverter under large load variations. Transactions on Power Delivery, 1, 1083-1087. 